\title{
Characterization of Mannheimia (Pasteurella) haemolytica leukotoxin interaction with bovine alveolar macrophage $\beta 2$ integrins
}

\author{
Praveen THUMBIKAT, Thamotharampillai DILEEPAN, \\ Mathur S. KANNAN, Samuel K. MAHESWARAN* \\ Department of Veterinary and Biomedical Sciences, University of Minnesota, St. Paul, \\ Minnesota 55108, USA
}

(Received 1 November 2004; accepted 21 March 2005)

\begin{abstract}
Mannheimia (Pasteurella) haemolytica, the etiologic agent of bovine pneumonic mannheimiosis, produces an exotoxic leukotoxin. The leukotoxin (LktA) is a member of the RTX (repeats in toxin) family of bacterial cytolysins and is distinguished from other toxins by its unique target cell specificity to ruminant leukocytes occurring through binding to a specific receptor. We have demonstrated previously that the $\beta_{2}$ integrin LFA-1 is a receptor for LktA in bovine leukocytes and is involved in leukotoxin-induced biological effects. However the subunits within LFA-1 involved in binding to LktA, and post-binding signaling leading to cellular activation have not been well characterized. The purpose of our study was to pinpoint these precise subunits on bovine alveolar macrophages and to characterize their interaction with LktA. The results in this study indicate that although LktA can efficiently bind to the CD18 subunit of both LFA-1 and Mac-1, post-binding signaling events including elevation of intracellular calcium and CD18 tail phosphorylation are only observed through LFA-1. Furthermore, LktA also binds to the CD11a subunit of LFA-1. LktA binding to CD11a could be inhibited by a small molecule inhibitor of the I(inserted)-domain, the major ligand binding interface on CD11a. I-domain inhibition significantly blunts LktA-induced intracellular calcium elevation and tyrosine phosphorylation of the CD18 tail. Based on our results we suggest that LFA-1 serves as the functional leukotoxin receptor on bovine alveolar macrophages.
\end{abstract}

Mannheimia haemolytica leukotoxin / receptor / lymphocyte function associated antigen-1 / signaling

\section{INTRODUCTION}

Mannheimia (Pasteurella) haemolytica serotype 1 is the primary bacterial agent of bovine pneumonic mannheimiosis commonly called shipping fever pneumonia. The disease is a cause of considerable eco- nomic loss to the beef and dairy cattle industries of North America [56]. The bacterium produces a variety of virulence factors [8], of these a large body of evidence indicates that the leukotoxin (LktA) and lipopolysaccharide (LPS) play a significant role in the pathogenesis that lead to the lung

* Corresponding author: mahes001@umn.edu 
pathology characteristic of the disease [59]. The LktA is a calcium dependent cytolytic protein belonging to the RTX (repeats in toxin) family of cytolytic proteins. Most RTX cytolysins are promiscuous and cause intoxication of a wide variety of vertebrate cells and display little or no cell-type or species specificity. In contrast, the leukotoxins produced by $A$. actinomycetemcomitans and $M$. haemolytica specifically interact with leukocytes from human and other primates $[43,46]$, and platelets and leukocytes from ruminant species respectively [4, 23]. With bovine leukocytes, at high concentrations, the LktA creates pores on the cell membrane leading to cellular swelling and lysis $[5,6]$. At sublytic concentrations, LktA activates bovine leukocytes and generates a myriad of inflammatory mediators $[4,7,10,11,13,17,36,37,44,45,58]$. The significance of LktA as a determinant of pathogenicity has been established in calf models with the use of genetically engineered isogenic leukotoxin deletion mutants of $M$. haemolytica [50].

Progress has been made in elucidating the molecular basis of the cell-type and species specific effects of $M$. haemolytica LktA. The narrow target cell specificity of LktA effects had led to the hypothesis that it acts through a specific receptor in ruminant leukocytes. In this regard, previous studies using bovine leukocytes have identified $\beta 2$ integrins as the receptors for LktA $[1,12,21,32,52]$. The $\beta_{2}$ integrins have a common $\beta$ subunit, the CD18 that associates with four distinct $\alpha$ subunits, CD11a, $\mathrm{CD} 11 \mathrm{~b}, \mathrm{CD} 11 \mathrm{c}$, and CD11d to give rise to four different $\beta_{2}$ integrins. They are: CD11a/CD18, also known as LFA-1 (lymphocyte function associated antigen-1); CD11b/CD18, also known as Mac-1 or CR3; CD11c/CD18, also known as P150/ 95 or CR4; and CD11d/CD18. The human LFA-1 subunits (CD11a and CD18) are products of separate genes [15, 18, 41, 47] and are expressed as heterodimers on the surface of all leukocytes. Each subunit is composed of a large extracellular domain $(\sim 1000$ residues for the $\alpha$-subunit and $\sim 750$ residues for the $\beta$-subunit), a single transmembrane domain of $\sim 20$ amino acids and a short cytoplasmic domain of $\sim 50$ amino acids. The $\alpha$ subunit contains in its aminoterminal third a 200-residue sequence [9], referred to as the I(inserted)-domain that is homologous to the A domain of von Willebrand factor [2]. This I-domain participates in ligand binding $[3,51]$ with mutations $[24,26,39]$ or deletions [28] in this domain resulting in abrogation of ligand binding and function. A number of studies have demonstrated that "outside in" LFA-1 activation and signaling are dependent on conformational changes induced in the I-domain as a result of interaction with a ligand [35, $38,48]$. These activation events, including phosphorylation of exposed tyrosine residues on the CD18 cytoplasmic tail and intracellular calcium elevation [16], have also been shown to be elicited in bovine leukocytes after exposure to LktA [22]. Stabilizing the I-domain in a closed conformation with a variety of statin-derived and non-statin small molecule compounds has been shown to allosterically inhibit ligand interaction and activation of LFA-1 [25, 53, 54]. Interestingly, studies with a related RTX toxin (LtxA) from A. actinomycetemcomitans suggest that the I-domain of human LFA-1 plays an important and critical role in the interaction with the toxin [27]. Antibodies mapped to epitopes on the I-domain of the human LFA-1 inhibit LtxA binding, suggesting a critical role for the LFA-1 I-domain.

While there is agreement that $\beta_{2}$ integrins are the receptors for LktA, there is controversy as to which $\beta_{2}$ integrin and its subunit functions as the functional receptor for LktA. Studies from another laboratory [1] indicated that the CD18 subunit of all three $\beta_{2}$ integrins served as the receptor for LktA. However, data generated from our laboratory using Western blot analysis of proteins eluted from an affinity chromatographybased binding assay and blocking experiments with anti- $\beta_{2}$ integrin monoclonal antibodies, concluded that LktA binds to CD11a and CD18, but not to CD11b or 
CD11c subunits [21]. These findings led us to suggest that the receptor for LktA is the CD11a subunit of LFA-1. These discordant findings from different laboratories underscore the need for additional studies to identify the precise receptor to which LktA binds and initiates signaling events in bovine leukocytes. Therefore, the objectives of the present study are to determine whether: (i) LktA binds to several $\beta 2$ integrins in bovine alveolar macrophages; (ii) binding occurs to the $\alpha$ and $\beta$ subunits of the $\beta 2$ integrins; and (iii) the signaling events that follow LktA binding require the interaction of the $\alpha$ and $\beta$ subunits of the $\beta 2$ integrins. To accomplish these objectives, we used bovine alveolar macrophages as target cells since these cells are uniquely positioned in the alveolar spaces for initial interaction with LktA and to initiate the inflammatory cascade in the disease.

\section{MATERIALS AND METHODS}

\subsection{Reagents and antibodies}

Dulbecco's modified Eagle's medium and RPMI 1640 were purchased from Celox Laboratories, Inc. (St. Paul, MN, USA). Fura-2/AM was purchased from Molecular Probes (Eugene, OR, USA). Polystyrene beads were obtained from Orange Products Inc. (Allentown, PA, USA). Phycoerythrin-labeled goat anti-mouse secondary antibodies were obtained from Jackson Immunoresearch (West Grove, PA, USA). Horseradish peroxidase-conjugated goat antimouse and anti-rabbit immunoglobulins $\mathrm{G}$ (IgG) were obtained from ICN Biomedical Research Products (Costa Mesa, CA, USA). Horseradish peroxidase-conjugated goat anti-mouse IgM was obtained from Pierce Chemical Co. (Rockford, Ill, USA). Molecular biology reagents were obtained from Invitrogen Co. (Carlsbad, CA, USA). All other reagents were obtained from Sigma Chemical Co. (St. Louis, MO, USA). The properties and applications of some of the various anti- $\beta 2$ integrin monoclonal anti- bodies (mAbs) used in the present study were previously described [21]. mAbs MM12A (anti-bovine CD11b), BAT75A (anti-bovine CD18), and BAQ30A (antibovine CD18) were purchased from VMRD Inc. (Pullman, WA, USA). mAbs R15.7 (anti-canine CD18) and R3.1 (anti-canine CD11a I-domain), R7.1 (anti-human CD11a I-domain), R3.3 (anti-human CD18 I-like domain) were a gift from R. Rothlein (Boehringer Ingelheim Pharmaceuticals Inc., Ridgefield, CT, USA). mAbs R3.I and R7.1 cross-react with the bovine I domain homologue, and R15.7 cross reacts with bovine CD18 [21]. IL-A99 (anti-bovine CD11a), IL-A130 (anti-bovine CD11b) and IL-A16 (anti-bovine CD11c) antibodies were provided by J. Naessens (ILRAD, Nairobi, Kenya). MD1H11 (anti-CD11a) was provided by J.J. Letesson (Facultés Universitaires Notre Dame de la Paix, Namur, Belgium), R7928 (anti-CD11b) was provided by C. Parkos (Emory University, GA, USA) and BAQ153A (anti-CD11c) was provided by W.C. Davis (Washington State University, Pullman, WA, USA). The antiLktA neutralizing mAb601 was a generous gift from S. Srikumaran (University of Nebraska, Lincoln, NE, USA). An irrelevant, isotype matched control $\mathrm{mAb}$ (MOPC21) was purchased from Sigma Chemical Co.

Lovastatin, a CD11a I domain inhibitor was purchased from Calbiochem (San Diego, CA, USA) and the statin-like I-domain antagonist LFA703 [53, 54] was kindly provided by Gabrielle Weitz-Schmidt (Novartis Pharma AG, Basel, Switzerland). BIRT377 [25, 57] another LFA-1 specific I-domain antagonist was provided by Terence A. Kelly (Boehringer Ingelheim Ridgefield, CT, USA). The inhibitors were diluted in assay buffer and always compared with a DMSO control where the same concentration of DMSO $(0.1 \%)$ as used in the highest inhibitor concentration was used.

\subsection{Primary cells and cell culture}

LktA-susceptible primary cells bovine alveolar macrophages (BAMs) were isolated 
from several 6- to 8-week-old healthy calves as described previously [21]. Viability of cells was determined by the trypan blue exclusion assay. For intracellular calcium $\left(\left[\mathrm{Ca}^{2+}\right]_{\mathrm{i}}\right)$ measurements, round $15-\mathrm{mm}$ diameter glass poly-L-lysine coated coverslips were plated with $300 \mu \mathrm{L}$ of a BAMs suspension in Dulbecco's modified Eagle's medium supplemented with $5 \%$ fetal bovine serum (FBS) containing of $7.5 \times 10^{5}$ cells $/ \mathrm{mL}$. The coverslips were placed inside tissue culture plates and incubated at $37{ }^{\circ} \mathrm{C}$ in a humidified atmosphere containing $5 \% \mathrm{CO}_{2}$. Cultured cells were used after 2 days of incubation. BAM cell lysates were prepared as previously described [21]. Briefly, $5 \times 10^{7}$ cells were suspended in $1 \mathrm{~mL}$ of lysis buffer $(\mathrm{pH} 7.5)\left(200 \mathrm{mM} \mathrm{NaCl}, 40 \mathrm{mM} \mathrm{NaHCO}{ }_{3}\right.$, $0.5 \%$ 3-[(3-cholamidopropyl)-dimethylammonio]-1-propanesulfonate (CHAPS), $10 \%$ glycerol, $1 \mathrm{mM}$ phenyl-methyl-sulfonyl fluoride, $5 \mu \mathrm{g}$ of leupeptin per $\mathrm{mL}, 5 \mu \mathrm{g}$ of pepstatin per $\mathrm{mL}, 0.01 \% \mathrm{NaN}_{3}$ ), incubated on ice, vortexed intermittently for $30 \mathrm{~min}$, and centrifuged at $100000 \times g$ at $4{ }^{\circ} \mathrm{C}$ for $1 \mathrm{~h}$. The supernatant containing the lysates was stored at $-80{ }^{\circ} \mathrm{C}$. Protein concentration of the lysates was measured with the DC-protein assay kit (Bio-Rad, Hercules, CA, USA). Immortal primary bovine lymphoma (BL-3) cells were grown in RPMI-1640 containing, $2 \mathrm{mM}$ L-glutamine, $100 \mathrm{U} / \mathrm{mL}$ penicillin and $100 \mu \mathrm{g} / \mathrm{mL}$ streptomycin. Cells were passaged at 1:3 ratios every other day.

\subsection{Preparation of $M$. haemolytica LktA}

Preparation and purification of native leukotoxin (LktA) from M. haemolytica wild D153 strain has been described previously [58]. The purity of the LktA was confirmed by sodium dodecyl sulfate-polyacrylamide gel electrophoresis (SDS-PAGE) and western blot analysis, and stored in the lyophilized state at $-20{ }^{\circ} \mathrm{C}$, and all studies were done with the same batch of LktA. The concentration of bioactive LktA was quantified by a colorimetric XTT assay [58] and was expressed as LktA units (LU) per mg dry weight. In order to exclude the effect of post-purification lipopolysaccharide (LPS) contamination, LktA was incubated with $10 \mu \mathrm{g}$ of polymyxin B per mL for $30 \mathrm{~min}$ on ice prior to use. LktA was biotinylated using EZ-Link Sulfo-NHS-LC-Biotin (Pierce Chemical Co., Rockford, Ill, USA) as per manufacturer's protocol, assayed for activity on BL-3 cells using the XTT assay and stored at $-70{ }^{\circ} \mathrm{C}$ till use.

\subsection{LktA-binding assays}

LktA binding to $\beta_{2}$ integrins in BAMs was examined in the presence or absence of antibodies or inhibitors. Anti- $\beta_{2}$ integrin antibodies or inhibitors at specific concentrations were added to BAMs, incubated for 15-30 min, and 50 LU per $\mathrm{mL}$ of biotinylated LktA was added. After an additional incubation for $45 \mathrm{~min}$ at $37{ }^{\circ} \mathrm{C}$, the cells were fixed with $1 \%$ paraformaldehyde (PFA) for 10 min and washed with FACS buffer. The cells were then incubated with streptavidin conjugated to phycoerythrin (SAPE) for $30 \mathrm{~min}$ on ice. Thereafter, the cells were washed and resuspended in $100 \mu \mathrm{L}$ of FACS buffer and fluorescence was analyzed by a FACS caliber flow cytometry system using CellQuest software (Becton Dickinson Immunoctometry Systems, San Jose, CA, USA). Background mean fluorescence intensity, MFI (without primary $\mathrm{mAb}$ ) was subtracted in all experiments. LktAbinding was expressed in terms of MFI and inhibition was calculated based on an LktA positive control and a negative control.

LktA binding to individual $\beta 2$ integrin subunits was examined using an affinity chromatography approach as described previously [21]. Briefly, polystyrene beads (0.125-in. diameter) were incubated with $2 \mathrm{~mL}$ of a solution of $20 \mu \mathrm{g}$ of purified LktA in PBS, $\mathrm{pH} 7.5$, overnight at $4{ }^{\circ} \mathrm{C}$ with gentle rocking. The beads were washed once with PBS and incubated with $1 \%$ bovine serum albumin (BSA) to block the remaining protein binding sites. Beads coated with $1 \%$ BSA served as a control. Bovine CD18 and residual heterodimers of CD11/CD18 
were sequentially depleted from BAM cell lysates, prepared as described earlier, by immunoprecipitation with BAT75A mAb. Depleted lysates were then diluted 1:3 with PBS containing $1 \mathrm{mM} \mathrm{CaCl} 2$ and $1 \mathrm{mM}$ $\mathrm{MgCl}_{2}$ and incubated with the LktA- or BSA-coated beads at $4{ }^{\circ} \mathrm{C}$ for $15 \mathrm{~h}$. The beads were then washed once with PBS, and the bound proteins were eluted from the beads by boiling with $50 \mu \mathrm{L}$ of SDS-PAGE loading buffer followed by Western blotting and probing with anti-CD11a (MD1H11), antiCD11b (R7928) or anti-CD11c (BAQ153A) antibodies, followed by detection with the appropriate horseradish peroxidase-conjugated secondary antibody. Expression levels of individual $\beta 2$ integrins was confirmed by immunoprecipitating CD11a, CD11b, and CD11c separately using IL-A99, IL-A130, IL-A16 mAbs. BAM cell lysate which was not depleted of CD18 and processed as described above in this section, served as a control to demonstrate LktA binding to CD18.

\section{5. $\beta_{2}$ integrin binding assay}

A modified affinity chromatography assay was used to demonstrate LktA binding to immobilized bovine LFA-1 or Mac-1. Anti-bovine CD11a (IL-A99), anti-bovine CD11b (IL-A130) or isotype control mAbs was coupled to Sepharose CL4B beads (Amersham) and used to immunoprecipitate LFA-1 and Mac-1 respectively from $100 \mu \mathrm{L}$ of precleared BAM lysates $(1 \times$ $10^{8}$ cells). The beads bound to the respective $\beta_{2}$ integrin were blocked with $1 \%$ BSA for $1 \mathrm{~h}$ followed by incubation overnight at $4{ }^{\circ} \mathrm{C}$ with LktA in HBSS containing $1 \mathrm{mM}$ $\mathrm{Ca}^{2+}$ and $\mathrm{Mg}^{2+}$. The bound LktA was cross linked with $1 \%$ PFA for 10 min to enhance stability. The beads were washed four times with HBSS followed by addition of Laemmli sample buffer containing $\beta$-mercaptoethanol. Eluted proteins were separated on 4-15\% SDS-PAGE gradient gels under non-reducing conditions and subjected to Western blotting as described previously. Thereafter, the membrane was incubated with 1:20 000 dilution of mAb601 (antiLktA) antibody for $1 \mathrm{~h}$ at room temperature followed by washes and incubation with a 1:50 000 dilution of the appropriate horseradish peroxidase-conjugated secondary antibody for $1 \mathrm{~h}$ at room temperature. The blots were developed using the SuperSignal ULTRA chemiluminescence detection system (Pierce Chemical Co.).

\section{6. $\left[\mathrm{Ca}^{2+}\right]_{i}$ measurement}

LktA-induced elevation of $\left[\mathrm{Ca}^{2+}\right]_{\mathrm{i}}$ level was measured by video fluorescence microscopy as described previously [19]. BAMs attached to poly-L-lysine coated glass coverslips were incubated in HBSS containing $2.5 \mathrm{mM} \mathrm{CaCl}_{2}, 1.2 \mathrm{mM} \mathrm{MgCl}_{2}$ and $5 \mu \mathrm{M}$ fura-2-acetoxymethyl ester (Fura-2/AM) for $30 \mathrm{~min}$ at $37^{\circ} \mathrm{C}$. The cells were then washed in HBSS, the coverslips were placed on the stage of a Diaphot inverted microscope (Nikon, Inc., Garden City, NY, USA), and the cells were exposed to LktA and viewed using a $40 \times$ fluor objective. The microscope was coupled to a digitally controlled filter wheel (DG-4; Sutter instrument Co., Novato, CA, USA), which contains filters for 340 and $380 \mathrm{~nm}$ excitation wavelengths. A photometric Cool Snap CCD 12-bit camera (Roper Scientific, Tucson, AR, USA) was used to measure fluorescence at an emission wavelength of $510 \mathrm{~nm}$. The output of the digital camera was sampled by a digital computer (Universal Imaging Corp., West Chester, PA, USA). To examine the effects of anti- $\beta 2$ integrin mAbs on LktAinduced $\left[\mathrm{Ca}^{2+}\right]_{\mathrm{i}}$ elevation, BAMs were preincubated with anti-CD11a, anti-CD11b, anti-CD11c, anti-CD18 or control mAb for $30 \mathrm{~min}$ at $37^{\circ} \mathrm{C}$ prior to LktA exposure. In some experiments where inhibitors were used, cells were pre-incubated with the inhibitor for $15 \mathrm{~min}$ prior to LktA exposure and collection of images. Fluorescence signals were acquired from regions of interest and images were corrected for system background, shading errors, and the autofluorescence of unloaded cells. The $\left[\mathrm{Ca}^{2+}\right]_{\mathrm{i}}$ 
concentrations were determined using a calibration method, as described [55]. The percent inhibition of $\left[\mathrm{Ca}^{2+}\right]_{\mathrm{i}}$ elevation was calculated using the formula described previously [21].

\subsection{Tyrosine phosphorylation of $\beta_{2}$ integrins}

LktA-induced tyrosine phosphorylation of the CD18 subunit tail of LFA-1 or Mac-1 on BAMs was assessed using the procedure that has been described previously [22]. In some experiments, BAMs were pretreated with inhibitors for $15 \mathrm{~min}$ at room temperature followed by LktA for $5 \mathrm{~min}$. Cells were lysed with buffer containing $1 \mathrm{mM}$ sodium orthovanadate. LFA-1 was immunoprecipitated from cell lysates using the mAb R7.1 (anti-human I domain) antibody coupled to protein A Sepharose CL-4B beads (Amersham Labs). In a separate experiment, Mac-1 was immunoprecipitated from cell lysates using mAb IL-A130. Immunoprecipitated proteins were separated on a 4-15\% SDS-gradient polyacrylamide gel and immunoblotted with an antiphosphotyrosine monoclonal antibody (mAb 4G10).

\subsection{Statistical analysis}

All results were expressed as means \pm standard error of means (SEMs). Comparisons were made with the one-way ANOVA test of significance between groups. Posttest comparisons among individual groups were done using Dunnett's multiple comparisons procedure. The term significant indicates a $P$ value of less than 0.05 . All tests were carried out using PRIZM software (Graphpad).

\section{RESULTS}

\subsection{LktA binds to CD18 of multiple $\beta 2$ integrins}

We examined the effects of mAbs directed against the CD18 subunit and the
Table I. Effect of anti- $\beta 2$ integrin antibodies on binding of biotinylated LktA to BAMs. Cells were pre-incubated with $5 \mu \mathrm{g}$ of various $\mathrm{mAbs}$ for $15-30 \mathrm{~min}$, followed by addition of $50 \mathrm{LU} /$ $\mathrm{mL}$ of LktA and incubation for an additional 45 min at $37^{\circ} \mathrm{C}$. Binding of biotinylated LktA to BAMs was determined by FACS analysis, and inhibition of binding was calculated as described in Materials and Methods. Inhibition of binding denoted in column three indicates significant $(* P<0.01)$ reduction in mean fluorescence intensity of bound biotinylated LktA on BAMs pretreated with anti- $\beta 2$ integrin antibodies.

\begin{tabular}{|c|c|c|}
\hline $\begin{array}{l}\beta 2 \text { integrin } \\
\text { antibodies }\end{array}$ & $\begin{array}{l}\text { Epitope } \\
\text { specificity }\end{array}$ & LktA binding \\
\hline \multicolumn{3}{|l|}{ (A) CD11a } \\
\hline i. R3.1 & I domain & No inhibition \\
\hline ii. R7.1 & I domain & No inhibition \\
\hline iv. IL-A99 & Not characterized & No inhibition \\
\hline \multicolumn{3}{|l|}{ (B) $\mathrm{CD} 11 \mathrm{~b}$} \\
\hline i. MM12A & Not characterized & No inhibition \\
\hline ii. R7928 & Not characterized & No inhibition \\
\hline iii. IL-A130 & Not characterized & No inhibition \\
\hline \multicolumn{3}{|l|}{ (C) $\mathrm{CD} 11 \mathrm{c}$} \\
\hline i. BAQ153A & Not characterized & No inhibition \\
\hline ii. IL-A16 & Not characterized & No inhibition \\
\hline \multicolumn{3}{|l|}{ (D) $\mathrm{CD} 18$} \\
\hline i. R3.3 & I-like domain & No inhibition \\
\hline ii. BAQ30A & Not characterized & Inhibition \\
\hline iii. BAT75A & Not characterized & Inhibition \\
\hline iv. R15.7 & Not characterized & Inhibition \\
\hline
\end{tabular}

various $\alpha$ subunits of the $\beta 2$ integrins on binding of biotinylated LktA to BAMs by FACS analysis. LktA binding to BAMs was inhibited by anti-CD18 mAbs BAQ30A, BAT75A and R15.7 (Tab. I). By contrast, R3.3, a mAb directed against the I-like domain of CD18, had no inhibitory effect on LktA binding to BAMs suggesting that the I-like domain of CD18 does not constitute the critical binding site for LktA. The mAbs directed against CD11a, CD11b and CD11c subunits of the $\beta 2$ integrins did not inhibit binding of LktA to BAMs suggesting that the $\alpha$ subunits do not play a critical 
role in LktA binding. Furthermore, antihuman CD11a I-domain mAbs R3.1 and R7.1 did not inhibit LktA binding. And, binding was abolished by preincubating biotinylated LktA with neutralizing antiLktA mAb601 (data not shown). Taken together, these results suggest that the $\alpha$ subunit is not critical for LktA binding to the CD18 subunit of $\beta 2$ integrins in BAM primary cells.

To further validate that the LktA binding epitope on BAMs is within the CD18 subunit, we tested the ability of LktA to bind both LFA-1 and Mac-1 in BAMs in a $\beta 2$ integrin binding assay using a modified affinity chromatography approach. Sepharose CL-4B beads coupled to immunoprecipitated LFA-1, Mac-1, or an isotypematched control $\mathrm{mAb}$ were incubated with LktA. The proteins from the beads were eluted and subjected to immunoblotting using anti-LktA mAb. Figure 1 shows that LktA binds to both the bovine LFA-1 and Mac-1, but not to any $\beta 2$ integrins bound to control mAb. The blots were stripped and reprobed with anti-LFA-1 or anti-Mac-1 mAbs to exclude the possibility of crosscontamination of Mac-1 immunoprecipitates with LFA-1 or vice versa (data not shown). The results of the binding experiments indicate that LktA binds to both bovine $\beta 2$ integrins.

\subsection{Effects of anti- $\beta 2$ integrin antibodies on LktA-induced $\left[\mathrm{Ca}^{2+}\right]_{\mathrm{i}}$ elevation}

We used elevation of $\left[\mathrm{Ca}^{2+}\right]_{i}$ as an index of activation because calcium ions are involved in the regulation of a diverse array of LktA-induced biological effects. We examined the effects of a panel of antibodies directed against CD18 and the three a subunits of bovine $\beta 2$ integrins on LktAinduced $\left[\mathrm{Ca}^{2+}\right]_{\mathrm{i}}$ elevation in BAMs. In the presence of anti-CD11a mAbs R7.1, R3.1 and IL-A99, there was significant inhibition of LktA-induced $\left[\mathrm{Ca}^{2+}\right]_{i}$ elevation (Fig. 2). By contrast, anti-CD11b mAbs

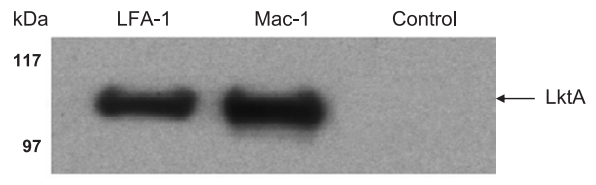

Figure 1. Western blot demonstrating binding of LktA to immobilized bovine LFA-1 and Mac-1 from BAMs. LFA-1 and Mac-1 were immunoprecipitated from BAMs with appropriate mAbs linked to Sepharose CL-4B beads and incubated separately with LktA, followed by detection of bound LktA with an anti-LktA $\mathrm{mAb}$. Note the control lane is BAM lysate proteins immunoprecipitated with an isotype matched control mAb, exposed to LktA, followed by western blotting, and probed with anti-LktA mAb. The data shown is representative of three independent experiments.

MM12A and IL-A130 or anti-CD11c mAb IL-A16 had no significant effects on LktAinduced $\left[\mathrm{Ca}^{2+}\right]_{\mathrm{i}}$ elevation (Fig. 2). AntiCD18 mAbs BAQ30A, BAT75A and R15.7 also significantly inhibited LktAinduced $\left[\mathrm{Ca}^{2+}\right]_{\mathrm{i}}$ elevation. The finding that anti-CD18 I-like domain mAb R3.3 significantly inhibited LktA-induced $\left[\mathrm{Ca}^{2+}\right]_{\mathrm{i}}$ elevation indicates that the I-like domain of CD18 plays a critical role in the signaling event leading to activation. Taken together, these results indicate that LktA-induced $\left[\mathrm{Ca}^{2+}\right]_{\mathrm{i}}$ elevation in BAMs requires the cooperation of CD11a and CD18 of LFA-1.

\subsection{LktA induces tyrosine phosphorylation of the CD18 tails of LFA-1 but not Mac-1}

Tyrosine phosphorylation of the CD18 tail was also used as a marker for the initial signaling event that follows binding of LktA to its receptor [21]. In this study, we immunoprecipitated bovine LFA-1 and Mac-1 from primary BAM lysates following pre-treatment of the cells with LktA. The immunoprecipitated LFA-1 and Mac1 were examined for tyrosine phosphorylation. The results show that LFA-1, but not the Mac-1, CD18 tail was tyrosine phosphorylated (Fig. 3). 


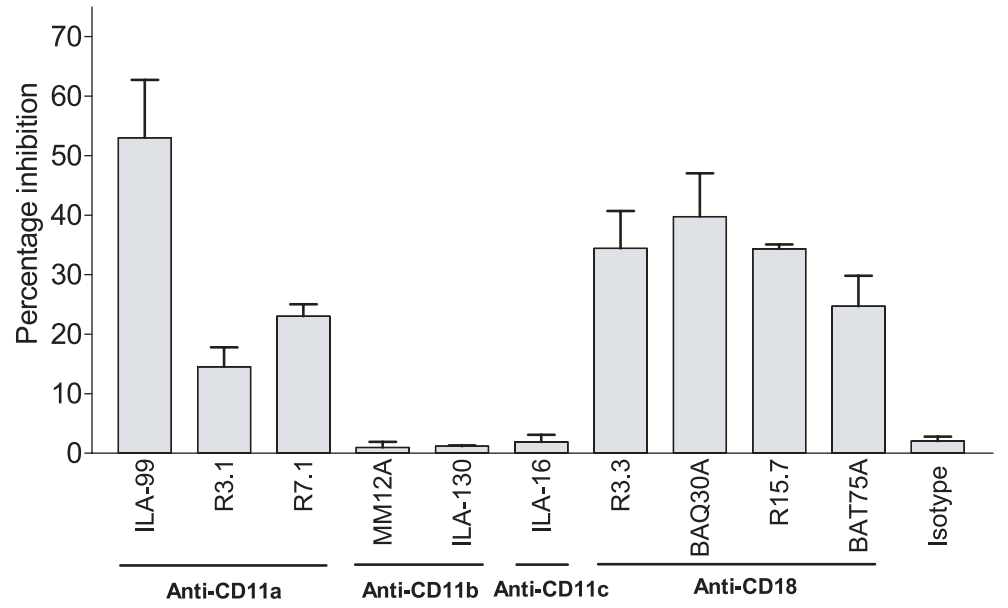

Figure 2. Effect of various anti- $\beta 2$ integrin antibodies on LktA-induced $\left[\mathrm{Ca}^{2+}\right]_{\mathrm{i}}$ elevation in BAMs. Cells were pre-incubated for $30 \mathrm{~min}$ at $37{ }^{\circ} \mathrm{C}$ with various mAbs at a concentration of $50 \mu \mathrm{g} \mathrm{per}$ $\mathrm{mL}$ followed by exposure to $50 \mathrm{LU} / \mathrm{mL}$ of LktA. The data shown are the mean $\pm \mathrm{SEM}$ from three independent experiments and expressed as the percentage of LktA-induced $\left[\mathrm{Ca}^{2+}\right]_{\mathrm{i}}$ elevation that is inhibited in the presence of the corresponding anti- $\beta 2$ integrin antibody. Note anti-CD11a and anti-CD18 but not anti-CD11b or anti-CD11c antibodies inhibit LktA-induced $\left[\mathrm{Ca}^{2+}\right]_{\mathrm{i}}$ elevation.

\subsection{LktA directly binds to the CD11a subunit of LFA-1}

In BAMs, LktA-induced activation requires both $\mathrm{CD} 18$ and $\mathrm{CD} 11 \mathrm{a}$, but not CD11b or CD11c, since only antibodies directed against the CD18 and CD11a subunits significantly inhibited LktA effects (see above). Therefore, we examined whether the LktA-induced effects also require binding to the $\alpha$ subunit of the $\beta 2$ integrins. Using lysis conditions designed to result in separation of $\beta 2$ integrin heterodimers into constituent monomers and to exclude binding to any residual heterodimers, cell lysates were sequentially depleted of CD18 by subjecting to immunoprecipitations prior to use in the binding assay. The results show that LktA binds to both the CD11a and CD18 subunits, but not to the CD11b or CD11c subunits (Fig. 4A).

The effects of CD11a I-domain inhibitors and anti-I-domain antibodies were used to ascertain the contribution of the CD11a I-domain on LktA binding. The

\section{LFA-1 Mac- 1}

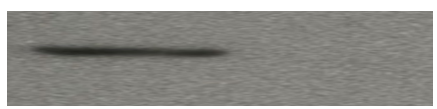

\section{CD18}

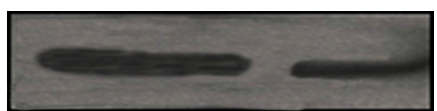

Figure 3. Western blot analysis of tyrosine phosphorylation of the CD18 tails of LFA-1 and of Mac-1 from BAMs. BAMs were exposed to $5 \mathrm{LU}$ of LktA for $5 \mathrm{~min}$ followed by preparation of cell lysates, immunoprecipitation of CD18, and immunoblotting with an anti-phosphotyrosine antibody (mAb 4G10). The membrane was stripped and reprobed with anti-CD18 mAb BAQ30A (lower panel). The data shown is representative of three independent experiments. CD18 tail of LFA-1, but not Mac-1 was tyrosine phosphorylated.

results show that the I-domain inhibitor BIRT377 significantly inhibited LktA binding to CD11a (Fig. 4B). However, BIRT377 as well as another I-domain inhibitor, LFA703, did not inhibit the binding of 
A.

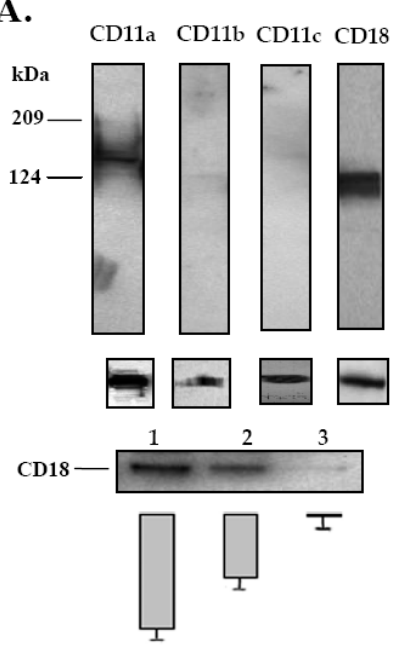

C.

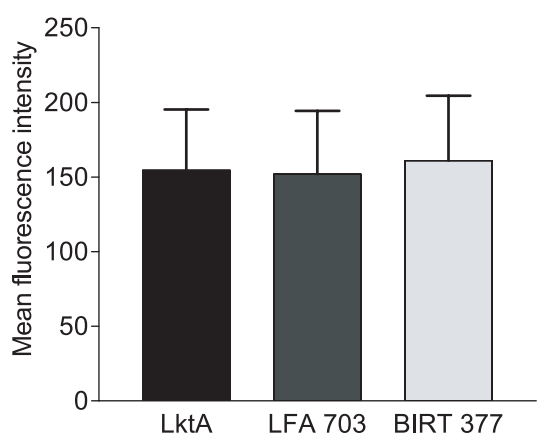

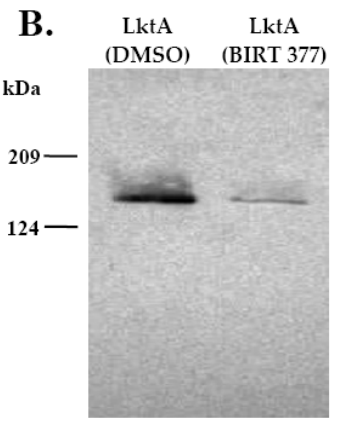

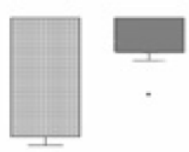

biotinylated LktA to BAMs (Fig. 4C). Similarly, the anti-I domain antibodies R3.1 and R7.1 inhibited LktA binding to CD11a, but not the binding of biotinylated LktA to BAMs (data not shown). These results demonstrate that the I domain inhibitors do not interfere with LktA binding to the CD18 subunit and that interaction of LktA with the CD11a subunit involves the I-domain.

\subsection{I domain inhibitors attenuate LktA-induced elevation of $\left[\mathrm{Ca}^{2+}\right]_{i}$ elevation in BAMs}

We examined the effects of the I-domain antagonists BIRT377, LFA703 and lovas-

Figure 4. Western blots demonstrating direct binding of LktA to CD11a and CD18 (panel A), and inhibition of LktA binding by the I-domain antagonist BIRT377 (panel B). Immunoprecipitated $\beta 2$ integrin subunit protein levels are shown beneath the corresponding LktA binding results in panel A. The sequential depletion of CD18 from cell lysates used for the assay as well as the changes in density of the corresponding bands is shown in the bottom of panel A. Bottom of panel $\mathbf{B}$ is densitometry showing inhibition of LktA binding to CD11a by $50 \mu \mathrm{M}$ BIRT377. The data shown is a representative of three independent experiments. The I-domain antagonists BIRT377 and LFA703 at $50 \mu \mathrm{M}$ concentration did not inhibit binding of biotinylated LktA to BAMs as determined by FACS analysis is shown in panel $\mathbf{C}$ and represents the mean \pm SEM of three independent experiments. tatin on LktA-induced $\left[\mathrm{Ca}^{2+}\right]_{\mathrm{i}}$ elevation in BAMs. At a concentration of $50 \mu \mathrm{M}$, all three inhibitors significantly attenuated LktA-induced elevation of $\left[\mathrm{Ca}^{2+}\right]_{i}$ (Fig. 5A). The inhibitory effects of LFA703 and BIRT377 were concentration-dependent (Fig. 5B). The inhibitory effect of lovastatin on $\left[\mathrm{Ca}^{2+}\right]_{\mathrm{i}}$ elevation was retained in the presence of mevalonic acid, thereby ruling out any non-specific effects due to competitive inhibition of 3-hydroxy-3'-methyl glutaryl coenzyme A (HMG CoA) (data not shown). Furthermore, LPS- induced $\left[\mathrm{Ca}^{2+}\right]_{\mathrm{i}}$ elevation was not inhibited by lovastatin, BIRT377 or LFA703 even at a concentration of $1 \mu \mathrm{g} / \mathrm{mL}$ (data not shown). This lack 


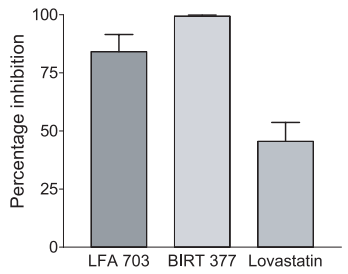

B
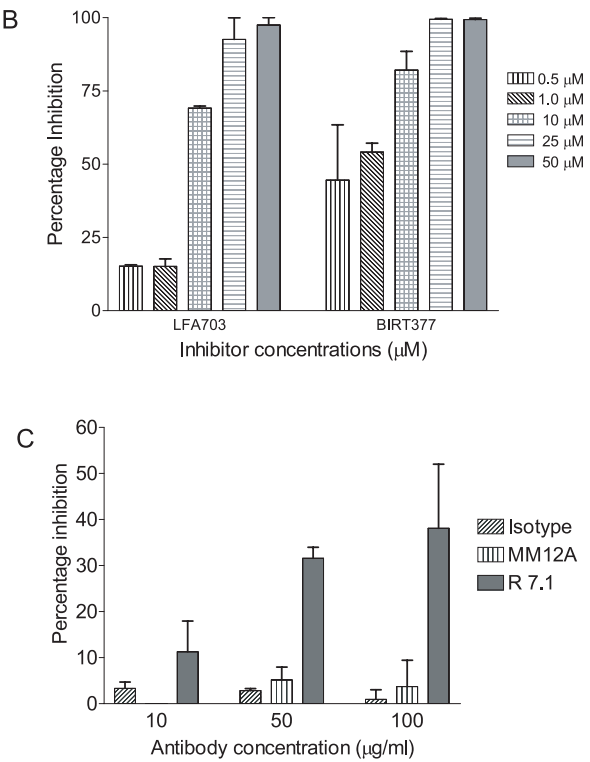

Figure 5. I-domain antagonists LFA703, BIRT377 and lovastatin at $50 \mu \mathrm{M}$ concentration inhibit LktA-induced $\left[\mathrm{Ca}^{2+}\right]_{\mathrm{i}}$ elevation in BAMs (A). The data shown represents the mean \pm SEM of three independent experiments. I-domain antagonists LFA703, BIRT377 (B) or an anti-I domain mAb R.7.1 (C) inhibits LktA-induced $\left[\mathrm{Ca}^{2+}\right]_{\mathrm{i}}$ elevation in BAMs in a concentrationdependent manner. The data shown represents the mean \pm SEM of three independent experiments.

of inhibition was observed over a range of different concentrations (data not shown), confirming that the inhibitory effects are specific to the LFA-1 signaling pathway.

The effects of the I-domain antagonists on LktA-induced $\left[\mathrm{Ca}^{2+}\right]_{\mathrm{i}}$ elevation in BAMs were validated using anti-I-domain antibodies. The anti-I-domain mAb R7.1

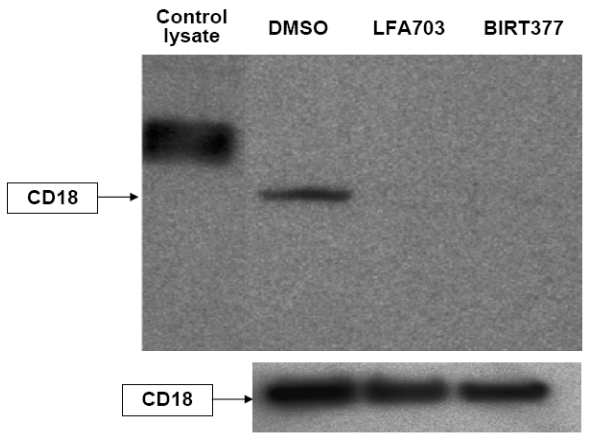

Figure 6. Western blots demonstrating the effect of I domain antagonists LFA703, and BIRT377 at $50 \mu \mathrm{M}$ concentration on LktAinduced tyrosine phosphorylation of the CD18 tail of LFA-1 from BAMs. The membrane was first developed with anti-phosphotyrosine antibodys (mAb 4G10), stripped, and reprobed with anti-CD18 mAb BAQ30A (lower panel). The data shown are representative of a set of three different experiments with the same results.

caused significant and concentration-dependent inhibition of LktA-induced $\left[\mathrm{Ca}^{2+}\right]_{i}$ elevation (Fig. 5C) as compared with antiCD11b mAb MM12A or an isotype control $\mathrm{mAb}$. These results suggest that the I-domain in CD11a of bovine LFA-1 is involved in LktA-induced $\left[\mathrm{Ca}^{2+}\right]_{\mathrm{i}}$ elevation in BAMs.

\subsection{I-domain antagonists inhibit LktA- induced phosphorylation of the cytoplasmic tail of bovine CD18}

We next examined the effects of LFA703 and BIRT377 on LktA-induced tyrosine phosphorylation of the CD18 cytoplasmic tail in BAMs. In the presence of LFA703 or BIRT377, there was significant inhibition of LktA-induced CD18 tail phosphorylation as compared to the DMSO vehicle control (Fig. 6). These results suggest that LktA-induced tyrosine phosphorylation of CD18 tails in BAMs, earlier shown to be a LFA-1 specific event (Fig. 3), requires $\mathrm{LktA}$-interaction with the I- domain of CD11a. 


\section{DISCUSSION}

The results of this study with bovine alveolar macrophages demonstrate unambiguously that LktA binds to the CD18 subunit of multiple $\beta 2$ integrins. However, post binding signaling events as shown by tyrosine phosphorylation of the CD18 tail occurs through LFA-1 but not Mac-1. We have also demonstrated that the I-domain of CD11a is critically involved in LktA signaling resulting in tyrosine phosphorylation of the CD18 tail and intracellular calcium elevation. This is the first report which describes discrete functions for the CD18 and CD11a subunits in the LFA-1/LktA interaction in bovine leukocytes.

Earlier investigations have attempted to identify the LktA receptor in bovine leukocytes and in heterologous systems in the context of its role in LktA-induced cytolysis. Of noteworthy, a study by Deshpande et al. had established using murine CD11a/ bovine CD18 chimeras that LktA-induced cytolysis is mediated by bovine CD18 suggesting that the receptor for LktA is CD18 [12]. However, their study did not examine the role of the $\alpha$ subunit of $\beta 2$ integrins in binding LktA and mediating other effects on target cells. In this study using BAMs as target cells, we demonstrate LktA binding to purified (by immunoprecipitation) LFA-1 and Mac-1 in BAMs (Fig. 1), suggesting that the CD18 is involved in such binding. This conclusion is further supported by the finding that antibodies directed against CD11a, CD11b, or CD11c do not inhibit LktA binding to BAMs, while anti-CD18 antibodies do (Tab. I). While these results apparently contradict an earlier report from our laboratory [21], the inhibition of LktA binding by human anti-CD11a mAbs demonstrated earlier using a different method may have resulted from steric hindrance of the CD18 binding sites. This is not a surprising finding since earlier reports by others have provided evidence for inhibition of ligand binding to receptors through steric hindrance by mAbs [33, 40, 42]. In the present study we have used an antibody directed against the bovine CD11a as opposed to the human anti-CD11a antibodies used in the previous study.

Previous work has shown that LktA interaction with bovine leukocytes causes an elevation in $\left[\mathrm{Ca}^{2+}\right]_{\mathrm{i}}[20] .\left[\mathrm{Ca}^{2+}\right]_{\mathrm{i}}$ is involved in the regulation of a diverse array of LktA-induced biological activity. This $\left[\mathrm{Ca}^{2+}\right]_{\mathrm{i}}$ elevation is required for NF- $\mathrm{KB}$ activation and proinflammatory gene expression [20], and has been shown to involve L-type $\mathrm{Ca}^{2+}$ channels in the plasma membrane [19]. We demonstrate that LktA-induced signaling in BAMs leading to $\left[\mathrm{Ca}^{2+}\right]_{i}$ elevation is mediated exclusively through the LFA-1 receptor. Our results confirm previous work by Jeyaseelan et al. demonstrating that LktA-induced $\left[\mathrm{Ca}^{2+}\right]_{\mathrm{i}}$ elevation can be specifically blocked using anti-CD11a antibodies but not with anti-CD11b or antiCD11c antibodies [21]. Anti-CD18 antibodies consistently blunted LktA-induced $\left[\mathrm{Ca}^{2+}\right]_{\mathrm{i}}$ elevation presumably due to inhibition of LktA binding to the CD18 subunit in the cell (Fig. 2). Taken together, these findings establishes a sequence of LktA interaction with BAMs, wherein binding of LktA to the CD18 subunit is required and the $\alpha$ subunit is critically involved in the signaling event leading to $\left[\mathrm{Ca}^{2+}\right]_{\mathrm{i}}$ elevation, and the signaling is mediated exclusively through LFA-1. The observation that CD18 is the LktA binding site, is compatible with the conclusions of other investigators [12, 32]. Our earlier conclusion [21] that CD11a is the binding site for LktA was based on the fact that LktA binding occurred to CD11a and CD18, but not CD11b or the CD11c subunits of other $\beta 2$ integrins. Furthermore, in the present study we demonstrate LktA binding to the CD11a subunit (Fig. 4A) and inhibition of this binding by the I-domain inhibitors (Fig. 4B). However, the I-domain inhibitors have no significant effect on LktA binding to the bovine LFA-1 (Fig. 4C) unlike mAbs directed against the CD18 subunit. The finding that antibodies directed against the I-like domain of CD18 did not inhibit LktA binding to BAMs, but blocked LktA-induced $\left[\mathrm{Ca}^{2+}\right]_{i}$ elevation indicates 
that although the binding site for LktA is not located in the I-like domain region of CD18, it plays a critical role in LktA-induced signaling leading to activation. These results suggest that CD18 subunit of bovine LFA-1 contains the LktA binding site and signaling through LFA-1 requires LktA interaction with the I-domain of CD11a, and this binding is critical in bringing about intersubunit interaction resulting in signaling.

An earlier communication from our laboratory reported that binding of LktA to its LFA-1 receptor induces a non-receptor tyrosine kinase signaling cascade [22]. This cascade results in tyrosine phosphorylation of the CD18 cytoplasmic tail and has been shown to specifically involve the Src and PI 3 kinases [22]. Inhibition of these kinases leads to attenuation of LktA-induced $\left[\mathrm{Ca}^{2+}\right]_{\mathrm{i}}$ elevation [22]. We therefore re-examined this issue by measuring the phosphorylation status of immunoprecipitated LFA-1 and Mac-1 from BAMs. Our results confirm that CD18 tail phosphorylation is specific to LFA-1 and does not occur in Mac-1 from bovine alveolar macrophages (Fig. 3).

We examined the role of the major ligand-binding domain of CD11a in both binding LktA and its biological effects. The I-domain in its human homolog has been shown to contain the metal-ion-dependent adhesion site (MIDAS), the major ligand binding interface on the CD11a subunit of LFA-1 [3, 51]. Since the results from studies from Fett et al. [14] and our independent study (GenBank accession no: AY382558) demonstrated a high degree of identity between the bovine CD11a I-domain and the human homologue at the cDNA sequence and predicted amino acid sequence levels ( $84.9 \%$ identity), we theorized that the bovine I-domain could serve as the major binding interface on CD11a for LktA. We also examined and verified using molecular modeling and I-domain antibody competition experiments that a human I-domain inhibitory ligand, lovastatin, was capable of binding the bovine I-domain and exerting an inhibitory effect on natural ligands (data not shown). Results from these studies suggested that the bovine and human I-domains are significantly conserved at the primary, secondary and tertiary levels and that an inhibitory ligand like lovastatin may be used to inhibit binding to the bovine CD11a subunit.

We therefore utilized lovastatin and the small molecule LFA-1 antagonists, BIRT377 and LFA703 to examine their effects on LktA interaction with bovine leukocytes. These antagonists have been characterized to bind to the human I-domain in and around the L-site and are known to abrogate ligand binding through maintaining LFA-1 in a closed conformation i.e. one unfavorable for ligand binding [53, 54, 57]. The effects of these antagonists are specific to LFA-1 and have been shown not to affect the other $\beta 2$ integrins from humans $[25,53]$ or bovine (data not shown). We show that BIRT377 inhibits LktA binding to bovine CD11a, suggesting the involvement of critical residues within the I-domain in LktA binding. In contrast, neither BIRT377 nor LFA703 inhibits the binding of biotinylated LktA to the CD18 subunit of BAMs. This finding was not surprising since the binding residues for LktA on BAMs reside on the CD18 subunit and would not be influenced by I-domain changes. In this regard, our results differ from those reported for the $A$. actinomycetemcomitans RTX toxin where overall binding to human leukocytes could be inhibited by an antibody mapped to the I-domain [27].

The role of the CD11a subunit in LktAinduced signaling events in BAMs was also examined. Inhibition of the I-domain using LFA703, BIRT377 or lovastatin significantly blunted LktA-induced $\left[\mathrm{Ca}^{2+}\right]_{\mathrm{i}}$ elevation (Fig. 5). This could be demonstrated in a concentration dependent manner and was verified using the anti-I-domain antibodies. These studies were extended to examine LktA-induced tyrosine phosphorylation of LFA-1 in BAMs pretreated with I-domain inhibitors (Fig. 6). The inhibition of both LktA-induced tyrosine phosphorylation 
and $\left[\mathrm{Ca}^{2+}\right]_{\mathrm{i}}$ elevation by these I-domain inhibitors strongly indicates the critical nature of the LktA-I-domain interaction in BAMs. Our results suggest that LktA-interaction with the I-domain of CD11a may be the distinguishing event that allows for signaling to proceed in case of LFA-1 but not in the case of the other $\beta 2$ integrins. These results are novel and have not been reported for any other RTX toxin. The apparent role for the I-domain of CD11a in LktA-induced signaling suggests that classical mechanisms of "outside-in" LFA-1 signaling may be activated within the target cell [54]. It is likely that well documented LFA-1 specific mechanisms including membrane raft formation [34], and cytokine mediated "insideout" signaling may also play a role in mediating LktA-induced biological effects. A recent report has in fact indirectly addressed this question and demonstrated that exposure of bovine neutrophils to IL-1ß, TNF- $\alpha$ and IFN- $\gamma$ increased the expression of LFA-1 leading to enhanced LktA effects [29]. These and other reports demonstrating that diverse ligands capable of eliciting intracellular signals that enhance LFA-1 mediated LktA-effects [29-31] suggest that LFA-1 affinity and avidity changes occurring through "inside-out" signaling may affect overall LktA biological effects on the target cell. It is very likely that binding of LktA to the I-domain of the CD11a subunit may confer, through allosteric interaction, a conformation to the CD18 subunit that is compatible for LktA signaling. The conclusion that LktA binds to the CD18 subunit of LFA-1 and Mac-1 does not in any way preclude CD18 of CR4 (CD11c/CD18) and CD11d/ CD18 as potential binding sites in BAMs, although this needs to be established.

The apparent ability of LktA to bind multiple $\beta 2$ integrins through the common CD18 subunit brings to mind a similar approach utilized by the Porphyromonas gingivalis fimbriae which binds multiple $\beta 2$ integrins on mouse peritoneal macrophages [49]. However, the $P$. gingivalis fimbriae utilize the $\alpha$ subunit for binding and exert functional effects through the $\beta$ subunit which appears to be the converse of the strategy involved in $M$. haemolytica LktA effects [49]. We propose that at LktA high concentrations, binding to the CD18 subunits of all three $\beta 2$ integrins mediates cellular cytotoxicity, presumably through a physical phenomenon leading to toxin oligomerization and pore formation. This allows an influx of extracellular $\mathrm{Ca}^{2+}$ and results in a sequence of cell damaging effects. LktA also directly interacts with the $\alpha$ subunit of LFA- 1 initiating a signaling cascade important for cellular activation. These activation events are likely to be of greater importance at lower concentrations of the toxin, known to result in activation [58] and apoptosis [44] as opposed to cytolysis. The majority of cellular pathology in bovine pneumonic mannheimiosis can be attributed to the overwhelming host immune response to the pathogen. Neutrophils, the major effector cells in the response to the pathogen, are recruited by the chemokines produced by activated alveolar macrophage to the site of injury and undergo activation and degranulation [11, 36]. This leads to production of pro-inflammatory mediators and the ensuing pneumonic events within the lung. Cellular activation mechanisms therefore play a central role in the pathogenesis of this disease. In the present study, we identify LFA-1 as the receptor mediating LktA-induced cellular activation and therefore propose that LFA-1 is the functional LktA receptor in bovine alveolar macrophages. Our study also describes discrete functions for the CD18 and CD11a subunits of LFA-1 in binding and activation. Our conclusion that CD18 subunit of LFA-1 contains the LktA binding site is based on the ability of mAbs to inhibit such binding. Final confirmation of the role or lack thereof of CD18 from the other $\beta 2$ integrins must await studies with recombinantly expressed bovine $\beta 2$ integrins in a heterologous system without the confounding effects of co-expression. However, these results have important implications in the design of therapeutic drugs aimed at inhibiting toxin effects. 


\section{ACKNOWLEDGEMENTS}

We are indebted to Shelia Alexander for help with flow cytometry analysis. We thank Dr Gabrielle Weitz-Schmidt (Novartis Pharma AG, Basel, Switzerland) for providing LFA703 and Dr Terrance A. Kelly (Boehringer Ingelheim Pharmaceuticals, Ridgefield, CT, USA) for providing compound BIRT377 used in this study.

\section{REFERENCES}

[1] Ambagala T.C., Ambagala A.P., Srikumaran S., The leukotoxin of Pasteurella haemolytica binds to beta(2) integrins on bovine leukocytes, FEMS Microbiol. Lett. 179 (1999) 161-167.

[2] Bienkowska J., Cruz J.M., Atiemo A., Handin R., Liddington R., The von Willebrand factor A3 domain does not contain a metal iondependent adhesion site motif, J. Biol. Chem. 272 (1997) 25162-25167.

[3] Calderwood D.A., Tuckwell D.S., Humphries M.J., Specificity of integrin I-domain-ligand binding, Biochem. Soc. Trans. 23 (1995) 504S-508S

[4] Clinkenbeard K.D., Upton M.L., Lysis of bovine platelets by Pasteurella haemolytica leukotoxin, Am. J. Vet. Res. 52 (1991) 453-457.

[5] Clinkenbeard K.D., Mosier D.A., Confer A.W., Transmembrane pore size and role of cell swelling in cytotoxicity caused by Pasteurella haemolytica leukotoxin, Infect. Immun. 57 (1989) 420-425.

[6] Clinkenbeard K.D., Mosier D.A., Timko A.L., Confer A.W., Effects of Pasteurella haemolytica leukotoxin on cultured bovine lymphoma cells, Am. J. Vet. Res. 50 (1989) 271-275.

[7] Clinkenbeard K.D., Clarke C.R., Hague C.M., Clinkenbeard P., Srikumaran S., Morton R.J., Pasteurella haemolytica leukotoxininduced synthesis of eicosanoids by bovine neutrophils in vitro, J. Leukoc. Biol. 56 (1994) 644-649.

[8] Confer A.W., Panciera R.J., Clinkenbeard K.D., Mosier D.A., Molecular aspects of virulence of Pasteurella haemolytica, Can. J. Vet. Res. 54 (1990) S48-S52.

[9] Curley G.P., Blum H., Humphries M.J., Integrin antagonists, Cell. Mol. Life Sci. 56 (1999) 427-441.

[10] Czuprynski C.J., Ortiz-Carranza O., Pasteurella haemolytica leukotoxin inhibits mitogen-induced bovine peripheral blood mononuclear cell proliferation in vitro, Microb. Pathog. 12 (1992) 459-463.

[11] Czuprynski C.J., Noel E.J., Ortiz-Carranza O., Srikumaran S., Activation of bovine neutrophils by partially purified Pasteurella haemolytica leukotoxin, Infect. Immun. 59 (1991) 3126-3133.

[12] Deshpande M.S., Ambagala T.C., Ambagala A.P., Kehrli M.E. Jr., Srikumaran S., Bovine CD18 is necessary and sufficient to mediate Mannheimia (Pasteurella) haemolytica leukotoxin-induced cytolysis, Infect. Immun. 70 (2002) 5058-5064.

[13] Dyer R.M., Benson C.E., Boy M.G., Production of superoxide anion by bovine pulmonary macrophages challenged with soluble and particulate stimuli, Am. J. Vet. Res. 46 (1985) 336-341.

[14] Fett T., Zecchinon L., Baise E., Desmecht D., The bovine (Bos Taurus) CD11a-encoding cDNA: molecular cloning, characterization and comparison with the human and murine glycoproteins, Gene 325 (2004) 97-101.

[15] Gahmberg C.G., Tolvanen M., Kotovuori P., Leukocyte adhesion: structure and function of human leukocyte $\beta 2$-integrins and their cellular ligands, Eur. J. Biochem. 245 (1997) 215-232.

[16] Garnotel R., Monboisse J.C., Randoux A. Haye B., Borel J.P., The binding of type I collagen to lymphocyte function-associated antigen (LFA) 1 integrin triggers the respiratory burst of human polymorphonuclear neutrophils. Role of calcium signaling and tyrosine phosphorylation of LFA 1, J. Biol. Chem. 270 (1995) 27495-27503.

[17] Henricks P.A., Binkhorst G.J., Drijver A.A., Nijkamp F.P., Pasteurella haemolytica leukotoxin enhances production of leukotriene B4 and 5-hydroxyeicosatetraenoic acid by bovine polymorphonuclear leukocytes, Infect. Immun. 60 (1992) 3238-3243.

[18] Hogg N.,Henderson R., Leitinger B., McDowall A., Porter J., Stanley P., Mechanisms contributing to the activity of integrins on leukocytes, Immunol. Rev. 186 (2002) 164-171.

[19] Hsuan S.L., Kannan M.S., Jeyaseelan S., Prakash Y.S., Sieck G.C., Maheswaran S.K., Pasteurella haemolytica A1-derived leukotoxin and endotoxin induce intracellular calcium elevation in bovine alveolar macrophages by different signaling pathways, Infect. Immun. 66 (1998) 2836-2844.

[20] Hsuan S.L., Kannan M.S., Jeyaseelan S., Prakash Y.S., Malazdrewich C., Abrahamsen 
M.S., Sieck G.C., Maheswaran S.K., Pasteurella haemolytica leukotoxin and endotoxin induced cytokine gene expression in bovine alveolar macrophages requires NFkappaB activation and calcium elevation, Microb. Pathog. 26 (1999) 263-273.

[21] Jeyaseelan S., Hsuan S.L., Kannan M.S., Walcheck B., Wang J.F., Kehrli M.E., Lally E.T., Sieck G.C., Maheswaran S.K., Lymphocyte function-associated antigen 1 is a receptor for Pasteurella haemolytica leukotoxin in bovine leukocytes, Infect. Immun. 68 (2000) 72-79.

[22] Jeyaseelan S., Kannan M.S., Briggs R.E., Thumbikat P., Maheswaran S.K., Mannheimia haemolytica leukotoxin activates a nonreceptor tyrosine kinase signaling cascade in bovine leukocytes, which induces biological effects, Infect. Immun. 69 (2001) 6131-6139.

[23] Kaehler K.L., Markham R.J., Muscoplat C.C., Johnson D.W., Evidence of species specificity in the cytocidal effects of Pasteurella haemolytica, Infect. Immun. 30 (1980) 615-616.

[24] Kamata T., Takada Y., Direct binding of collagen to the I domain of integrin alpha 2 beta 1 (VLA-2, CD49b/CD29) in a divalent cationindependent manner, J. Biol. Chem. 269 (1994) 26006-26010.

[25] Kelly T.A., Jeanfavre D.D., McNeil D.W., Woska J.R.J., Reilly P.L., Mainolfi E.A., Kishimoto K.M., Nabozny G.H., Zinter R., Bormann B.J., Rothlein R., Cutting edge: a small molecule antagonist of LFA-1-mediated cell adhesion, J. Immunol. 163 (1999) 5173-5177.

[26] Kern A., Briesewitz R., Bank I., Marcantonio E.E., The role of the I domain in ligand binding of the human integrin alpha 1 beta 1 , J. Biol. Chem. 269 (1994) 22811-22816.

[27] Lally E.T., Kieba I.R., Sato A., Green C.L., Rosenbloom J., Korostoff J., Wang J.F., Shenker B.J., Ortlepp S., Robinson M.K., Billings P.C., RTX toxins recognize a beta2 integrin on the surface of human target cells, J. Biol. Chem. 272 (1997) 30463-30469.

[28] Leitinger B., Hogg N., Effects of I domain deletion on the function of the beta2 integrin lymphocyte function-associated antigen-1, Mol. Biol. Cell 11 (2000) 677-690.

[29] Leite F., O'Brien S., Sylte M.J., Page T., Atapattu D., Czuprynski C.J., Inflammatory cytokines enhance the interaction of Mannheimia haemolytica leukotoxin with bovine peripheral blood neutrophils in vitro, Infect. Immun. 70 (2002) 4336-4343.

[30] Leite F., Gyles S., Atapattu D., Maheswaran S.K., Czuprynski C.J., Prior exposure to
Mannheimia haemolytica leukotoxin or LPS enhances beta(2)-integrin expression by bovine neutrophils and augments LKT cytotoxicity, Microb. Pathog. 34 (2003) 267-275.

[31] Leite F., Kuckleburg C., Atapattu D., Schultz R., Czuprynski C.J., BHV-1 infection and inflammatory cytokines amplify the interaction of Mannheimia haemolytica leukotoxin with bovine peripheral blood mononuclear cells in vitro, Vet. Immunol. Immunopathol. 99 (2004) 193-202.

[32] Li J., Clinkenbeard K.D., Ritchey J.W., Bovine CD18 identified as a species specific receptor for Pasteurella haemolytica leukotoxin, Vet. Microbiol. 67 (1999) 91-97.

[33] List K., Hoyer-Hansen G., Ronne F., Dano K., Behrendt N., Different mechanisms are involved in the antibody mediated inhibition of ligand binding of the urokinase receptor: a study based on biosensor technology, J. Immunol. Methods 222 (1999) 125-133.

[34] Lub M., van Kooyk Y., Figdor C.G., Ins and outs of LFA-1, Immunol. Today 16 (1995) 479-483.

[35] Lupher M.L. Jr., Harris E.A., Beals C.R., Sui L.M., Liddington R.C., Staunton D.E., Cellular activation of leukocyte function-associated antigen- 1 and its affinity are regulated at the I domain allosteric site, J. Immunol. 167 (2001) 1431-1439.

[36] Maheswaran S.K., Weiss D.J., Kannan M.S., Townsend E.L., Reddy K.R., Whiteley L.O., Srikumaran S., Effects of Pasteurella haemolytica A1 leukotoxin on bovine neutrophils: degranulation and generation of oxygenderived free radicals, Vet. Immunol. Immunopathol. 33 (1992) 51-68.

[37] Majury A.L., Shewen P.E., The effect of Pasteurella haemolytica A1 leukotoxic culture supernate on the in vitro proliferative response of bovine lymphocytes, Vet. Immunol. Immunopathol. 29 (1991) 41-56.

[38] McDowall A., Leitinger B., Stanley P., Bates P.A., Randi A.M., Hogg N., The I domain of integrin leukocyte function-associated antigen-1 is involved in a conformational change leading to high affinity binding to ligand intercellular adhesion molecule 1 (ICAM-1), J. Biol. Chem. 273 (1998) 27396-27403.

[39] Michishita M., Videm V., Arnaout M.A., A novel divalent cation-binding site in the $\mathrm{A}$ domain of the beta 2 integrin CR3 (CD11b/ CD18) is essential for ligand binding, Cell 72 (1993) 857-867.

[40] Nason E.L., Wetzel J.D., Mukherjee S.K., Barton E.S., Prasad B.V., Dermody T.S., A monoclonal antibody specific for reovirus 
outer capsid protein $\sigma 3$ inhibits $\sigma 1$-mediated hemagglutination by steric hindrance, $\mathrm{J}$ Virol. 75 (2001) 6625-6634.

[41] O’Toole T.E., Katagiri Y., Faull R.J., Peter K., Tamura R., Quaranta V., Loftus J.C., Shattil S.J., Ginsberg M.H., Integrin cytoplasmic domains mediate inside-out signal transduction, J. Cell Biol. 124 (1994) 1047-1059.

[42] Petersen H.H., Hansen M., Schousboe S.L., Andreasen P.A., Localization of epitopes for monoclonal antibodies to urokinase-type plasminogen activator: relationship between epitope localization and effects of antibodies on molecular interactions of the enzyme, Eur. J. Biochem. 268 (2001) 4430-4439.

[43] Shenker B.J., Vitale L.A., Keiba I., Harrison G., Berthold P., Golub E., Lally E.T., Flow cytometric analysis of the cytotoxic effects of Actinobacillus actinomycetemcomitans leukotoxin on human natural killer cells, J. Leukoc. Biol. 55 (1994) 153-160.

[44] Stevens P.K., Czuprynski C.J., Pasteurella haemolytica leukotoxin induces bovine leukocytes to undergo morphologic changes consistent with apoptosis in vitro, Infect. Immun. 64 (1996) 2687-2694.

[45] Sun Y., Clinkenbeard K.D., Clarke C., Cudd L., Highlander S.K., Dabo S.M., Pasteurella haemolytica leukotoxin induced apoptosis of bovine lymphocytes involves DNA fragmentation, Vet. Microbiol. 65 (1999) 153-166.

[46] Taichman N.S., Simpson D.L., Sakurada S., Cranfield M., DiRienzo J., Slots J., Comparative studies on the biology of Actinobacillus actinomycetemcomitans leukotoxin in primates, Oral Microbiol. Immunol. 2 (1987) 97-104.

[47] Takagi J., Springer T.A., Integrin activation and structural rearrangement, Immunol. Rev. 186 (2002) 141-163.

[48] Takagi J., Petre B.M., Walz T., Springer T.A., Global conformational rearrangements in integrin extracellular domains in outsidein and inside-out signaling, Cell 110 (2002) 599-611.

[49] Takeshita A., Murakami Y., Yamashita Y., Ishida M., Fujisawa S., Kitano K., Hanazawa S., Porphyromonas gingivalis fimbriae use beta2 integrin (CD11/CD18) on mouse peritoneal macrophages as a cellular receptor, and the CD18 beta chain plays a functional role in fimbrial signaling, Infect. Immun. 66 (1998) 4056-4060.

[50] Tatum F.M., Briggs R.E., Sreevatsan S., Zehr E.S., Ling Hsuan S., Whiteley L.O., Ames T.R., Maheswaran S.K., Construction of an isogenic leukotoxin deletion mutant of Pas- teurella haemolytica serotype 1: characterization and virulence, Microb. Pathog. 24 (1998) 37-46.

[51] Tuckwell D., Calderwood D.A., Green L.J., Humphries M.J., Integrin alpha 2 I-domain is a binding site for collagens, J. Cell Sci. 108 (1995) 1629-1637.

[52] Wang J.F., Kieba I.R., Korostoff J., Guo T.L., Yamaguchi N., Rozmiarek H., Billings P.C., Shenker B.J., Lally E.T., Molecular and biochemical mechanisms of Pasteurella haemolytica leukotoxin-induced cell death, Microb. Pathog. 25 (1998) 317-331.

[53] Weitz-Schmidt G., Welzenbach K., Brinkmann V., Kamata T., Kallen J., Bruns C., Cottens S., Takada Y., Hommel U., Statins selectively inhibit leukocyte function antigen-1 by binding to a novel regulatory integrin site, Nat. Med. 7 (2001) 687-692.

[54] Welzenbach K., Hommel U., Weitz-Schmidt G., Small molecule inhibitors induce conformational changes in the I domain and the I-like domain of lymphocyte function-associated antigen-1. Molecular insights into integrin inhibition, J. Biol. Chem. 277 (2002) 1059010598 .

[55] White T.A., Kannan M.S., Walseth T.F., Intracellular calcium signaling through the cADPR pathway is agonist specific in porcine airway smooth muscle, FASEB J. 17 (2003) 482-484.

[56] Whiteley L.O., Maheswaran S.K., Weiss D.J., Ames T.R., Kannan M.S., Pasteurella haemolytica A1 and bovine respiratory disease: pathogenesis, J. Vet. Intern. Med. 6 (1992) 11-22.

[57] Woska J.R. Jr., Last-Barney K., Rothlein R., Kroe R.R., Reilly P.L., Jeanfavre D.D., Mainolfi E.A., Kelly T.A., Caviness G.O., Fogal S.E., Panzenbeck M.J., Kishimoto T.K., Giblin P.A., Small molecule LFA-1 antagonists compete with an anti-LFA-1 monoclonal antibody for binding to the CD11a I domain: development of a flowcytometry-based receptor occupancy assay, J. Immunol. Methods 277 (2003) 101-115.

[58] Yoo H.S., Rajagopal B.S., Maheswaran S.K., Ames T.R., Purified Pasteurella haemolytica leukotoxin induces expression of inflammatory cytokines from bovine alveolar macrophages, Microb. Pathog. 18 (1995) 237-252.

[59] Zecchinon L., Fett T., Desmecht D., How Mannheimia haemolytica defeats host defense through a kiss of death mechanism, Vet. Res. 36 (2005) 13-25. 